\title{
Visual impressions of interactions between objects when the causal object does not move
}

\author{
Peter A White \\ School of Psychology, Cardiff University, Tower Building, Park Place, Cardiff CF10 3AT, UK: \\ e-mail:whitepa@cardiff.ac.uk \\ Received 6 August 2001, in revised form 1 October 2004; published online 13 April 2005
}

\begin{abstract}
Stimuli were presented that consisted of a stationary row of black-bordered white rectangles. As observers watched, each rectangle in turn from left to right changed from white to black. The final rectangle did not change colour but moved off from left to right. The sequential colour change suggested motion from left to right, and observers reliably reported a visual impression that this illusory motion kicked or bumped the last rectangle, thereby making it move. The impression was stronger when the sequential colour change was faster, but was not significantly affected by the number of the rectangles in the row (ranging from 2 to 8 ). These results support the conclusion that neither contact nor motion of a causal object is necessary for a visual impression of causality to occur.
\end{abstract}

\section{Introduction}

Imagine a visual display consisting of two small black rectangles, $\mathrm{A}$ and $\mathrm{B}$, in a field of uniform white. A moves towards B in a straight horizontal line at constant velocity. When A contacts B, A stops at the point of contact and B immediately moves off in the same direction at the same, or a slightly lower, speed. There is no obvious reason to perceive these motions as anything more than what they are: just two moving objects. The stimulus is sufficiently ambiguous to support several possible interpretations: object $\mathrm{B}$ might move of its own accord when contacted by A, like a runner in a relay race, or $\mathrm{A}$ might lie in a different plane and not contact $\mathrm{B}$ at all. Despite this ambiguity, people reliably perceive an interaction between the objects. Specifically, they perceive A kick, push, or launch B, thereby making it move. They perceive B not moving of its own accord, but made to move by A. This illusory causal impression, termed the launching effect, was first reported by Michotte (1946) and has been confirmed by many investigations since then (Beasley 1968; Boyle 1960, 1975; Costall 1991; Gordon et al 1990; Leslie 1982, 1984; Leslie and Keeble 1987; Michotte and Thinès 1991; Natsoulas 1961; Powesland 1959; Schlottmann and Shanks 1992; Scholl and Tremoulet 2000; White 1988, 1995; Yela 1952).

Early investigations employed an ingenious mechanical apparatus, described in full by Michotte (1963). Thick black lines were painted on a revolving disc. The disc was obscured apart from a $5 \mathrm{~mm}$ slit aligned along a radius of the disc. Parts of the black lines visible in the slit gave the subjective impression of rectangles. Painting the lines in spiral patterns yielded continuous translation of the apparent rectangle across the slit when the disc was rotated, giving an impression of motion that was fluent and realistic. More recent computers have been used to generate stimulus presentations as a series of frames projected in sequence on a monitor screen (eg Schlottmann and Shanks 1992; White and Milne 1999). Each frame is a static image. Frames succeed each other at the screen refresh rate, typically around $75 \mathrm{~Hz}$. At this rate, successive changes in the positions of objects in the frames appear as movements from one place to another (Hochberg and Brooks 1996). This apparent motion also appears fluent and realistic.

Gordon et al (1990) investigated stimuli in which the motion of A or B or both was in the form of phi movement. Phi movement of one object was achieved by 
presenting two identical images in sequence, $93 \mathrm{~mm}$ apart, on a computer screen. Initially, only one image was visible, then that image disappeared and the other image appeared. Three test stimuli were used: one with phi movement of A and real movement of $\mathrm{B}$, one with real movement of $\mathrm{A}$ and phi movement of $\mathrm{B}$, and one with phi movement of both. 'Real' movement means apparent motion produced by computer in the manner described in the previous paragraph. Figure 1 in Gordon et al (1990) shows that contact occurred between the objects in these stimuli. To investigate how the stroboscopic stimuli were perceived, Gordon et al used a magnitude-estimation procedure. In this, a standard launching-effect sequence with 'real' movement was assigned a strength of 10 and a sequence in which the onset of the motion of $\mathrm{B}$ was delayed for $1 \mathrm{~s}$ was assigned a strength of 0 . This latter sequence does not give rise to an impression of causality (Gordon et al 1990; Michotte 1963). When motion of both $\mathrm{A}$ and $\mathrm{B}$ was stroboscopic, a mean rating of 12.8 was given, suggesting that the sequence gave rise to a causal impression at least as strong as that generated by the launching stimulus with 'real' movement, possibly even stronger. When there was phi movement of just one object, ratings were significantly lower but still high enough to indicate the occurrence of a causal impression.

The illusory causal impression therefore occurs with each of the three types of apparent motion described. In fact, the launching effect is extraordinarily robust in that it occurs in perception of stimuli that are radically incomplete as representations of real interaction events. In the typical launching-effect stimuli used by Michotte (1963) and subsequent researchers, the objects are identifiable only as two-dimensional arrays of edges and angles - orientation with respect to the observer is uncertain, and the surrounding area is blank, lacking any cues to the context or the nature of the objects and their interaction. In some of Michotte's (1963) experiments, and in a more systematic investigation by Yela (1952), the launching effect was not appreciably diminished by the presence of a substantial gap between the two objects, which, of course, could never happen in real interactions between objects (except in such cases as magnetic repulsion). With a different kind of stimulus, observers reliably report a visual impression that one object is pulling one or more other objects, despite the lack of any visible connection between them (Michotte 1963; White and Milne 1997).

In view of the fact that these causal impressions occur in the absence of conditions that might be thought necessary for the occurrence of a real causal relation, namely contact in the case of the launching effect and connection in the case of the pulling impression, it is natural to ask just how incomplete a stimulus can be and still give rise to a visual interaction impression. This question is important because it places constraints on explanatory hypotheses. At the very least, any proposed model would have to explain how radically incomplete stimuli can be perceived as specific kinds of interactions between objects. In the present research, I investigate the possibility that a causal impression can still occur when the causal object does not move at all.

The technique involved presenting a row of stationary objects and implying motion by means of rapid sequential colour change. The basic stimulus is depicted in figure 1 . Figure la shows the first frame of the stimulus. Eight rectangles are arranged in a row below and to the left of centre. To the right of these rectangles is a square: this corresponds to object B in a standard launching-effect stimulus. After a brief interval, the left-hand rectangle turns black; after another brief interval, the one next to it turns black; and each rectangle in turn does the same, the intervals being the same throughout. Figure $1 \mathrm{~b}$ shows a frame from partway through this sequence. When the rectangle adjacent to the square turns black, the square immediately moves to the right at a constant speed of $13.5 \mathrm{~cm} \mathrm{~s}^{-1}$. Figure 1c shows the first frame of the motion of the square. Figure 1d shows a later frame. The square eventually exits the right side of the frame. 


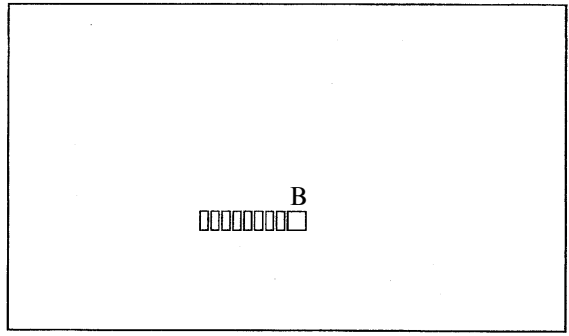

(a)

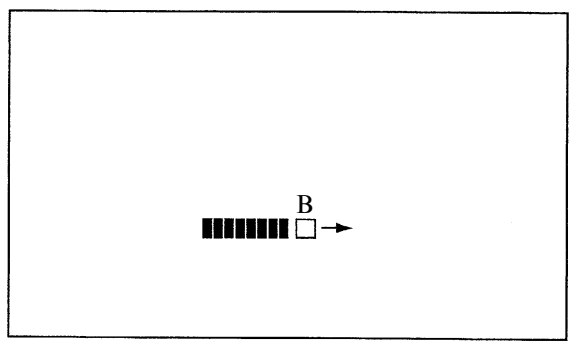

(c)

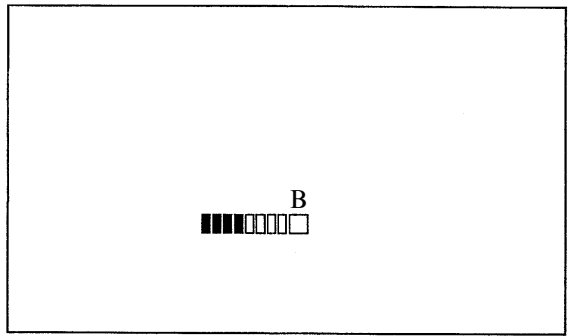

(b)

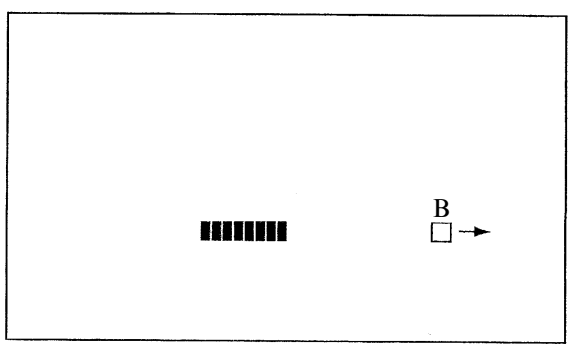

(d)

Figure 1. Schematic representation of four frames from the basic stimulus sequence used in the experiment. (a) Stimulus at start of sequence, with eight white rectangles and one square (object B, as labelled). (b) Frame partway through sequence, after four rectangles have sequentially turned black, starting at the left-hand side. (c) First frame of the motion of the square. (d) Later frame, prior to the square, B, exiting the right side of the frame.

This is different from the stimuli used by Gordon et al (1990). Rectangles do not appear and disappear, but merely change from white bounded by black lines to completely black. There are several rectangles, and the colour change occurs in sequence across the row. None of the rectangles moves and none is in contact with the square. One might expect that the square would be perceived as moving off of its own accord, on the grounds that none of the other rectangles moves or does anything other than change colour, and nothing contacts or is in contact with the square.

On the other hand, the sequential colour change evokes a strong impression of left-to-right motion. Moreover, there is exact temporal contiguity between the last rectangle turning black and the square starting to move. Pilot work and observations suggest that the stimulus yields a strong causal impression: an impression that the square is pushed or kicked into motion. The aim of the experiment was to investigate the reliability of this impression and the extent to which it depends on the number of rectangles visible and the delay between successive objects changing colour.

\section{Method}

\subsection{Participants}

The participants were thirty-eight first-year undergraduate students of subjects other than psychology, participating in return for cash payment.

\subsection{Apparatus and stimulus materials}

Stimuli consisted of frame sequences generated by a Power Macintosh 6100/60 computer and displayed on a Macintosh 16 inch colour monitor. The frames were presented in phase with the vertical blank signal of the computer, and appeared at the refresh rate of $74 \mathrm{~Hz}$. Each frame was 500 pixels $(18.5 \mathrm{~cm})$ wide by 300 pixels $(11.1 \mathrm{~cm})$ high. The boundaries of the frame appeared on the screen as thin black lines. These disappeared between stimulus presentations, leaving the screen uniform white. To achieve the necessary presentation rate, frames employed monochrome graphics. All sequences 
consisted of 200 frames, lasting $2.7 \mathrm{~s}$. The background of each frame was uniform white throughout the experiment.

The basic stimulus is depicted in figure 1. This figure presents actual frames from the stimulus, though reduced in scale in the process of printing and photocopying, and with arrows added to indicate the occurrence and direction of motion.

Two variables were manipulated. Two, four, six, or eight rectangles (other than object B) were visible. Figure 1 shows the eight-rectangle condition. Delay between successive rectangles turning black was either $54 \mathrm{~ms}$ (4 frames), $108 \mathrm{~ms}$ ( 8 frames), $162 \mathrm{~ms}$ (12 frames), or $216 \mathrm{~ms}$ (16 frames). These delay conditions were called short, fairly short, fairly long, and long, respectively.

\subsection{Procedure}

The experiment was run by an experimenter who was blind to the aims and hypotheses of the experiment. Participants were tested individually in a small windowless laboratory, empty except for the equipment used for the experiment, with fluorescent lighting giving a low ambient-light level. Participants were seated so that their faces were approximately $75 \mathrm{~cm}$ from the screen and were permitted to adjust this distance slightly for personal comfort.

The experimenter introduced the experiment by telling the participants that they were going to see some stimuli that created a kind of visual illusion. Although nothing actually moved, it might look as though something was moving across the screen from left to right. The experimenter then presented a stimulus similar to figure 1, except that the square was absent. The experimenter asked the participant if he/she had the impression of movement across the screen from left to right. At this stage the experimenter had been primed with a procedure to use if the participant said "no" or expressed uncertainty, but in fact all participants immediately reported that they did have the impression of movement.

The experimenter then told the participants that they were going to see movies that showed stimuli roughly similar to the one they had just seen. In these stimuli, however, they would see an object actually move off the end of the line. The experimenter then presented a stimulus similar to that shown in figure 1, with a delay immediate between the fast and fairly fast delays used in the experimental stimuli. While the stimulus was being presented, the experimenter called the object that moved object B. She then told the participant that, although nothing actually contacted object $\mathrm{B}$, the participant might have the impression that the illusory movement somehow bumped or pushed B, thus making it move. This suggestion was made overtly because of a concern that participants might be reluctant to report a causal impression when no moving object contacted B, as a result of evaluation apprehension or similar concerns. The experimenter was again primed with a procedure to use if the participant said he/she had no causal impression or expressed any kind of uncertainty, but in fact all participants reported the causal impression with no uncertainty.

The experimenter then said that, for each of the movies that followed, the participants' task was to report how strong an impression of bumping or pushing they had. They were given a written response sheet with a series of trial numbers, and by each trial number was the question: "How strong a causal impression did you have that time?". The participants were instructed to write a number from 0 to 100 by the question, where 100 meant that they had a very strong impression of bumping or pushing and 0 meant that they had no such impression. The stronger the impression they had, the higher the number they should put.

When the participants indicated that they were ready the experimental trials began. Trials were presented with the use of an automated procedure controlled 
by the participant. Before the first trial, a sign appeared on the screen saying "sequence 1". Under this was a button tagged with the label "show". Participants were instructed that, when they were ready to see the first trial, they should use the mouse to click on the "show" button, and the stimulus would be shown immediately. When the "show" button was clicked, the computer ran the trial. After a short pause a sign came up saying "sequence 2" with the "show" button beneath it. When participants had filled out the rating for the first trial and were ready to continue, they clicked on the "show" button and the next trial was presented. In this way, participants progressed through the experimental trials at their own pace. The experimenter watched from a distance and did not intervene. The order of trials was randomised by the computer independently for each participant, and the order of trials used for a given participant was retrieved from the computer by the experimenter after the run was complete. At the end of the run participants were thanked, paid, and debriefed.

\section{Results}

Ratings were analysed with a 2 (number of rectangles, two versus four versus six versus eight $) \times 2$ (delay, short versus fairly short versus fairly long versus long) analysis of variance with repeated measures on both factors. A significant main effect of delay was found $\left(F_{3,111}=56.27, p<0.001\right)$. A posteriori comparisons revealed the order short $(75.43)>$ fairly short $(64.92)>$ fairly long $(51.18)>$ long (46.84) (all differences $p<0.05)$. The main effect of number of objects was not statistically significant $\left(F_{3,111}=1.34\right)$. There was, however, a significant interaction between the two factors $\left(F_{9,333}=5.93, p<0.001\right)$ which is depicted in figure 2 .

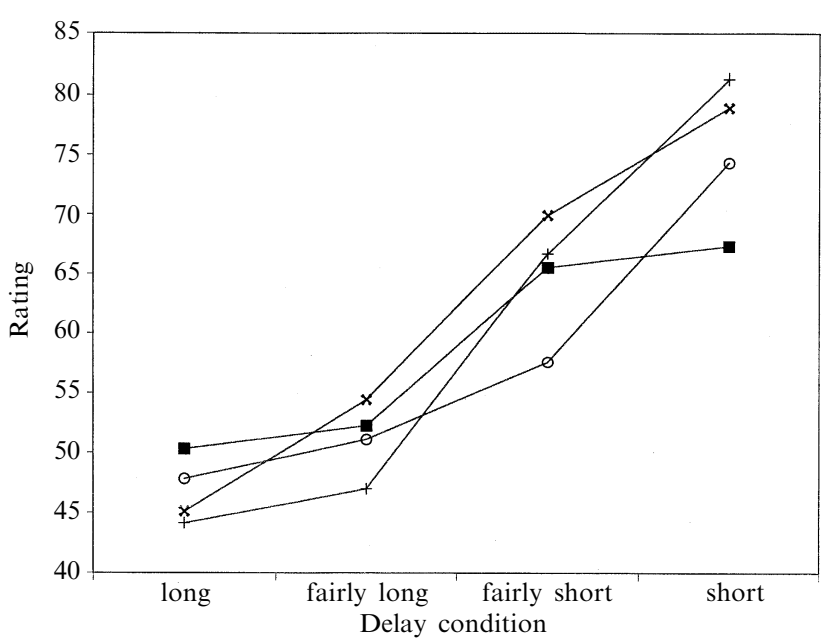

$$
\begin{aligned}
& \text { Number of rectangles } \\
& \text { - two } \\
& \text { - four } \\
& \text { * six } \\
& + \text { eight }
\end{aligned}
$$

Figure 2. Interaction between delay condition and number of rectangles comprising object $\mathrm{A}$ in launching-effect stimuli.

The figure shows that the main effect of length of delay dominates the results. However, the slope of the curves is greatest with eight objects and least with two, suggesting that increasing the number of objects increasingly differentiates the perceptual impression.

One aim of this experiment was to investigate the reliability of the causal impression. Figure 3 presents the frequency distribution for 10-point ranges of the rating scale of causal impression for the stimulus with the highest mean rating (short delay with eight objects). The figure clearly shows a high degree of consensus, with all but three participants rating the causal impression of the stimulus 70 or higher, and none lower than 50 . 


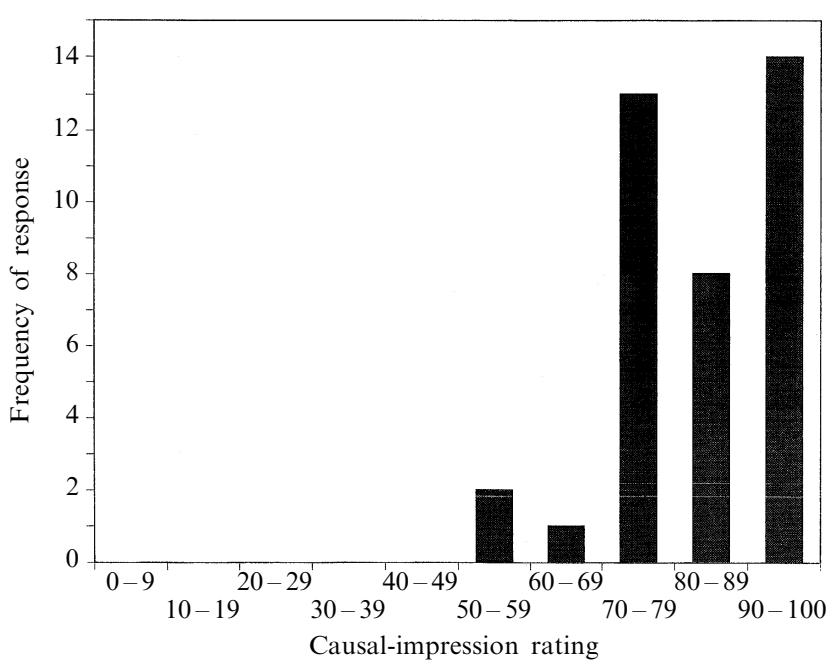

Figure 3. Frequency distribution of participants' causal-impressionrating responses for the stimulus with fast delay and with eight rectangles comprising object $\mathrm{A}$.

\section{Discussion}

In this experiment, strong causal impressions were reported under conditions that might be thought unlikely to give rise to such impressions. A series of rectangles underwent a sequential colour change from white to black. No rectangle moved, the sequence of changes was slow enough to be tracked comfortably by eye, and between the rectangles there were visible gaps that underwent no change. Object B moved off to the right when the last rectangle turned black; again, these two objects were never in contact. All participants reported a strong impression of left-to-right motion and, particularly in conditions where the delay between successive colour changes was $54 \mathrm{~ms}$, a strong impression that the illusory movement somehow bumped or pushed the square, thus making it move.

The procedure conveys some idea of my state of mind before the experiment was run. I was uncertain whether participants would have any impression of left-to-right movement or that they would understand what the experimenter meant when she referred to it. I was doubly uncertain whether they would report any causal impression or that they would understand what the experimenter meant when she referred to that. One reason for this doubt was that a fair proportion of naïve observers fail to report the launching effect with standard launching stimuli, such as those used by Michotte (1963) (Boyle 1960; Gemelli and Cappellini 1958; White 1988). It did not seem likely, therefore, that a degraded version of a launching-effect stimulus would yield consistent reports of a causal impression. In fact, these doubts were groundless and all participants immediately confirmed both the impression of left-to-right motion and the causal impression. Figure 3 indicates that the causal impression is particularly reliable with these stimuli.

Against this, it could be argued that the procedure primed participants to perceive an interaction because the experimenter made explicit mention of this possibility before the experimental trials began. The possibility that ratings may have been inflated by such priming cannot be entirely ruled out, but I would argue that its effects are no more than minimal. Past studies have similarly primed participants by using a particular form of wording in a rating scale: an example is the measure of pulling employed by White and Milne (1997). However, for some stimuli in that research, uniformly low ratings of pulling were obtained - in one case as low as 11.30 on a 101-point scale. This supports the claim that if participants do not experience the interaction impression they do not give high ratings on the scale, irrespective of any priming effect the 
instructions or scale wordings might have. In addition, priming effects would apply uniformly to all stimuli and therefore do not explain the significant effects of the experimental manipulations obtained here.

With standard launching-effect stimuli, the causal impression is strongest when the speed of object B is the same as or slightly less than that of A before contact (Michotte 1963; Natsoulas 1961). If B moves faster than A, the launching effect disappears and observers instead have the impression that the movement of $\mathrm{B}$ was 'triggered' by A (Michotte 1963). This term ("déclenchement" in the original-Michotte 1946) refers to an impression that $\mathrm{A}$ has touched off the movement of $\mathrm{B}$, which then appears to move of its own accord. It is, as the description implies, qualitatively different from launching.

In the present stimuli, the square moved at a speed of $13.5 \mathrm{~cm} \mathrm{~s}^{-1}$. The sequential changes of the rectangles from white to black in the short-delay condition happened at a rate that was equivalent to a speed of $7.82 \mathrm{~cm} \mathrm{~s}^{-1}$. In the long-delay condition, the rate was equivalent to a speed of $1.95 \mathrm{~cm} \mathrm{~s}^{-1}$. These speeds are much slower than that of the square, and Michotte's findings would suggest that the launching effect should not occur under these conditions. Yet in the short-delay condition with eight rectangles, the reported causal impression was very strong, as figure 3 shows. Moderate ratings were obtained even in the long-delay conditions.

One possible explanation for this result is that the causal impression that occurs in perception of these stimuli is not the same as the launching effect. Without employing Michotte's methods of experimental phenomenology it is impossible to be certain. The high ratings consistently given to some of the stimuli in experiment 1 imply that descriptions in terms of bumping and pushing are considered suitable to the phenomenal impression, which suggests at least a close affinity to the launching effect and rules out triggering, as described by Michotte (1963). The possibility of a triggering impression at long delay cannot be ruled out, but the primary concern here is to account for the stimuli that gave rise to the highest ratings.

In my experience, the causal impression generated by the present experimental stimuli has an almost explosive quality, as if the square is a projectile launched by a cannon or by a grenade exploding nearby, although not quite as explosive as that. Of course, I cannot vouch for the participants in the study in this respect. The impression generated by the launching-effect stimulus lacks that explosive quality and is, in my experience, harder to pin down to any definite character (see also Schlottmann 2000). Although the actual speed implied by the rate at which the rectangles turn black is comparatively slow, it does not look slow. It is therefore possible that the impression is strong because the difference in speed between the two phases of the stimulus is not perceived. Moreover, although the whole of the last rectangle turns black in 1 frame, as near instantaneously as the technology can manage, one almost has the feeling of an extremely rapid left-to-right transition of colour across the rectangle, as if it were a window through which the passage of a very rapidly moving object was discerned. This impression of very rapid motion could account for the explosive quality of the causal impression.

White and Milne $(1997,1999)$ have advocated a template-matching model of visualinteraction impressions (see also Dittrich and Lea 1994). In essence, observers acquire from visual experience a collection of stored representations of interaction events. These templates are somewhat abstracted and do not preserve full information about every experience that contributed to them, but could still be large in number. The features of a stimulus tend to activate the template that most closely matches the stimulus, and this template then functions as an interpretation of the stimulus. This is a fast and automatic process. In its interpretational function, the template specifies certain features of the event that are not present in the stimulus. The interaction impression is one of these. 
One attraction of the template-matching hypothesis is that it readily accommodates findings that visual interaction impressions occur with radically incomplete stimuli. This is most easily illustrated by the pulling impression (White and Milne 1997). In a typical stimulus for pulling, five rectangles are arranged in a column. The top rectangle starts moving from right to left at constant speed. After a short interval the second rectangle does the same, then the third, and so on. Observers reliably report an impression that the top rectangle pulls the others (White and Milne 1997). Under the template-matching account, the stimulus matches the features of a template of pulling events better than the features of any other kind of template. The application of the template in the perceptual interpretation of the stimulus yields the perceptual impression of pulling. The fact that no connection between the objects is visible does not prevent the stimulus from being interpreted as a pulling relation, so long as the visible features match the pulling template better than any other template. If this is the case, then the presence of an unseen connection is part of the perceptual interpretation of the stimulus, supplied, like the pulling impression, by the template.

To apply this explanatory account to the present results it is therefore necessary to identify a real-world circumstance that would give rise to a template against which the stimulus could be matched. The kind of real-world circumstance that appears most similar to our stimuli is the motion of an object perceived through a series of apertures, for example a fence made of separated palings or a window with several vertical bars close together. Motion of an object viewed through such a fence or window is normally perceived as continuous despite the repeated visual obstructions. I therefore propose that the sequential colour change of the rectangles matches the template for a single object moving behind a stationary object, such as a fence, that presents a series of apertures through which the moving object is viewed, and does not match any other template as well as that. The launching effect then occurs because the sequence is perceptually interpreted as a moving object striking a stationary object and setting it in motion. The explosive quality of the impression results from the lack of delay between the last rectangle changing colour and object B starting to move. This is consistent with an event in which the moving object abruptly accelerates at the end of its trajectory, thereby delivering a more powerful blow to object $\mathrm{B}$.

One theoretical alternative to template matching is the kinematic specification of dynamics (KSD) (Runeson 1983; Runeson and Frykholm 1981; Runeson et al 2000; Runeson and Vedeler 1993). According to KSD, observers pick up the dynamics of an event when the dynamics are unambiguously specified by the kinematic information. This is the case in two-object collisions such as the typical launching-effect stimulus. Runeson (1983) showed that the velocities of two objects after collision are uniquely determined under the laws of mechanics by their velocities before collision, their masses, and the coefficient of restitution, and that, by rearrangement, the (relative) masses of the objects are specified by their velocities before and after collision and by the coefficient of restitution. Studies have shown that observers can make very accurate estimates of dynamical properties, such as relative mass, from kinematic information alone (Runeson and Frykholm 1981; Runeson et al 2001; Runeson and Vedeler 1993; Todd and Warren 1982).

It is doubtful whether KSD can account for the present results, however. There are two reasons for this. One is that dynamic properties can only be specified when there is a collision and motion of both objects is perceived. In the present case there is no collision and the causal object does not move, so the available kinematic information is insufficient to construct the dynamical properties of the objects.

The second reason for doubting the capacity of KSD to explain these results is that inferences of mass ratios and visual interaction impressions are different things. 
The launching effect is not an impression of the relative masses of the objects, but an impression that a specific kind of interaction occurs between them. The qualitatively distinct nature of this impression is revealed most readily by comparison: it is unequivocally qualitatively different from pushing (White and Milne 1997), enforced disintegration (White and Milne 1999), and entraining (Michotte 1963), for example. Even if it is hard to pin down the qualitative characteristics of the launching impression, it is clear that they are distinct from those of these other interaction impressions. Runeson (1983) dismissed the launching effect on grounds of vagueness, by comparison with the multiple factors that determine outcomes of collision events in the laws of mechanics. But it is not vague by comparison with other kinds of interaction impressions: it is just not the same as inferred dynamical properties.

Some studies have shown that, with stimuli in which objects move without colliding, observers tend to interpret the motion of the objects as animate. One object may be seen, for example, as fleeing from another (Blythe et al 1999; Heider and Simmel 1944; Michotte 1963; Tremoulet and Feldman 2000). In the present stimuli there is no contact between the objects, so could the motion of object $\mathrm{B}$ be perceived as animate motion? The instructions for the causal rating scale explicitly referred to an impression of object $\mathrm{A}$ bumping or pushing object $\mathrm{B}$, and the high ratings given on this measure for many of the stimuli show that this description captures the visual impressions of many of the participants. In other words, even though there is no collision, they still perceive the motion of B to be caused by A bumping it. This is not an impression of animate motion. Lack of contact may be necessary for an impression of animate motion, but as the present findings and the pulling impression (White and Milne 1997) both demonstrate, it is not sufficient for it.

There are other possible accounts of visual impressions of interactions between objects, including Michotte's (1963) Gestalt account and the hypothesis that visual impressions of causality are the work of innate visual mechanisms that are activated by particular stimulus configurations (Leslie and Keeble 1987; Scholl and Tremoulet 2000). These may also be able to account for the findings. The Gestalt account, in particular, may be able to explain the impression of motion from left to right. The rapid sequential colour change fits the principle of good continuation of motion, provided one accepts that the gaps between the rectangles do not disrupt good continuation. Good continuation was a central feature of Michotte's (1963) theoretical account of the launching effect, so the possible role of that principle in determining the impression of motion in perception of the present stimuli appears to be compatible with Michotte's account.

However the present findings should be interpreted, they are a novel and striking example of the way in which visual impressions of causality can occur in perception of stimuli that are radically incomplete or inadequate as representations of real interactions between objects. The very occurrence of the launching effect in perception of nothing more than geometrical shapes and kinematic patterns is remarkable enough. The occurrence of the effect in stimuli in which the causal object neither moves nor contacts the other object is an impressive testament to the robustness of the phenomenon.

Acknowledgments. The software used to construct the stimuli was designed by Alan Milne. I am grateful to Sophie Connolly for acting as experimenter and to Lorraine Awcock for preparing figure 1 .

\section{References}

Beasley N E, 1968 "The extent of individual differences in the perception of causality" Canadian Journal of Psychology $22399-407$

Blythe P W, Todd P M, Miller G F, 1999 "How motion reveals intention: categorizing social interactions", in Simple Heuristics that Make Us Smart Eds G Gigerenzer, P M Todd, the ABC Research Group (Oxford: Oxford University Press) pp 257-285 
Boyle D G, 1960 "A contribution to the study of phenomenal causality" Quarterly Journal of Experimental Psychology 12171 - 179

Boyle D G, 1975 "A new technique for studying phenomenal causality" British Journal of Psychology $66383-385$

Costall A, 1991 "Phenomenal causality", in Michotte's Experimental Phenomenology of Perception Eds G Thinès, A Costall, G Butterworth (Hove, East Sussex: Lawrence Erlbaum Associates) pp $51-64$

Dittrich W H, Lea S E G, 1994 "Visual perception of intentional motion" Perception 23 253-268

Gemelli A, Cappellini A, 1958 "The influence of the subject's attitude in perception" Acta Psychologica $1412-23$

Gordon I E, Day R H, Stecher E J, 1990 "Perceived causality occurs with stroboscopic movement of one or both stimulus elements" Perception 19 17-20

Heider F, Simmel M, 1944 "An experimental study of apparent behavior" American Journal of Psychology $57243-259$

Hochberg J, Brooks V, 1996 "The perception of motion pictures", in Cognitive Ecology Eds M P Friedman, E C Carterette (London: Academic Press) pp 205-292

Leslie A M, 1982 "The perception of causality in infants" Perception 11 173-186

Leslie A M, 1984 "Spatiotemporal continuity and the perception of causality in infants" Perception $13287-305$

Leslie A M, Keeble S, 1987 "Do six-month-old infants perceive causality?" Cognition 25 265-288

Michotte A, 1946 La Perception de la Causalité (Louvain: Institut Supérieur de Philosophie)

Michotte A, 1963 The Perception of Causality (New York: Basic Books)

Michotte A, Thinès G, 1991 "Perceived causality", in Michotte's Experimental Phenomenology of Perception Eds G Thinès, A Costall, G Butterworth (Hove, East Sussex: Lawrence Erlbaum Associates) pp 66-87

Natsoulas T, 1961 "Principles of momentum and kinetic energy in the perception of causality" American Journal of Psychology $74394-402$

Powesland P F, 1959 "The effect of practice upon the perception of causality" Canadian Journal of Psychology 13 155- 168

Runeson S, 1983 "On visual perception of dynamic events" Acta Universitatis Upsaliensis: Studia Psychologica Upsaliensia $91-56$

Runeson S, Frykholm G, 1981 "Visual perception and lifted weight" Journal of Experimental Psychology: Human Perception and Performance $7733-740$

Runeson S, Juslin P, Olsson H, 2000 "Visual perception of dynamic properties: cue heuristics versus direct-perceptual competence" Psychological Review 107 525-555

Runeson S, Vedeler D, 1993 "The indispensability of precollision kinematics in the visual perception of relative mass" Perception \& Psychophysics 53 617-632

Schlottmann A, 2000 "Is perception of causality modular?" Trends in Cognitive Sciences 4 441-442

Schlottmann A, Shanks D R, 1992 "Evidence for a distinction between judged and perceived causality" Quarterly Journal of Experimental Psychology A 44321 - 342

Scholl B J, Tremoulet P D, 2000 "Perceptual causality and animacy" Trends in Cognitive Sciences $4299-309$

Todd J T, Warren W H, 1982 "Visual perception of relative mass in dynamic events" Perception $11325-335$

Tremoulet P D, Feldman J, 2000 "Perception of animacy from the motion of a single object" Perception $29943-951$

White P A, 1988 "Causal processing: origins and development" Psychological Bulletin 104 36-52

White P A, 1995 The Understanding of Causation and the Production of Action: From Infancy to Adulthood (Hove, East Sussex: Lawrence Erlbaum Associates)

White P A, Milne A, 1997 "Phenomenal causality: impressions of pulling in the visual perception of objects in motion" American Journal of Psychology 110 573-602

White P A, Milne A, 1999 "Impressions of enforced disintegration and bursting in the visual perception of collision events" Journal of Experimental Psychology: General 128 499-516

Yela M, 1952 "Phenomenal causation at a distance" Quarterly Journal of Experimental Psychology $4139-154$ 


\section{PERCEPTION}

VOLUME 342005

www.perceptionweb.com

Conditions of use. This article may be downloaded from the Perception website for personal research by members of subscribing organisations. Authors are entitled to distribute their own article (in printed form or by e-mail) to up to 50 people. This PDF may not be placed on any website (or other online distribution system) without permission of the publisher. 